\title{
You can't have it all
}

\section{Compromise is a major unifying thread in the fabric of contemporary thought.}

\section{Neil S. Greenspan}

A nyone contemplating the transformation in scientific understanding over the past ten centuries would have to agree that progress has come on numerous and diverse fronts. Is there a conceptual link between these various advances that also distinguishes them from the notions of the past? In subjects as varied as logic, physics, biology and political theory, the acceptance of a fundamental role for limits has grown while the once automatic faith in absolutes has declined. Consequently, an intriguing candidate for a hallmark of modern thinking is the unassuming notion of the tradeoff, or compromise.

A logical starting point for considering this thesis is mathematical logic. Kurt Gödel's incompleteness theorem of 1931 established that no finite system of axioms could entail all of the truths that pertain to the arithmetic of natural numbers. To have the deductive power to prove all such truths, one requires additional axioms that inevitably permit the derivation of false statements pertaining to the natural numbers. Therefore, one can have deductive completeness or one can have logical consistency, but one can't have both in an axiomatization of arithmetic.

In physics, trade-offs, although of different sorts, figure in two of the greatest revolutions of the twentieth century. Although special relativity does offer us an absolute the speed of light in a vacuum - the consequence is the loss of an absolute frame of reference and the elimination of absolute simultaneity. Shifting focus from the cosmic to the sub-microscopic, Werner Heisenberg achieved fame beyond science by proposing that there is an inevitable trade-off between precision in the knowledge of a particle's position and precision in the knowledge of that particle's momentum. It is from his experience with this quantum realm that Niels Bohr derived his related idea of complementarity. The gist of Bohr's notion is that two perspectives can both be necessary to fully comprehend an entity or phenomenon and yet be mutually incompatible with respect to the experimental means and conditions required for their acquisition.

This notion of complementarity can be useful even at higher levels of organizational complexity. Modern biology is dominated by the effort to relate molecular structure to function. But to determine molecular structure typically requires molecular homogeneity. In contrast, the function of a gene product, or the gene that encodes it, is only derivable from the full range of its interactions with its heterogeneous chemical environment. As Max Delbrück, one of the
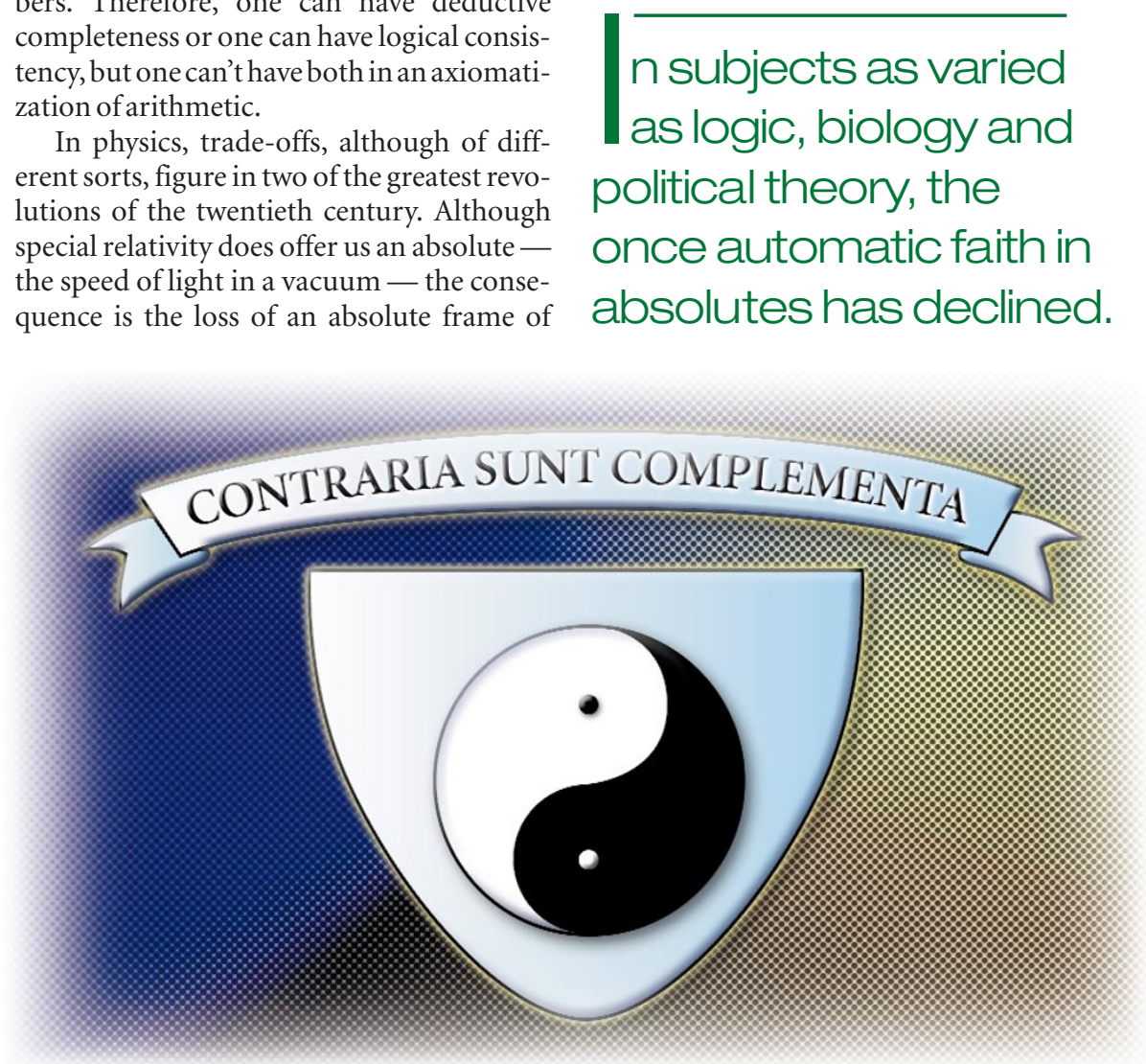

Quite contrary: Niels Bohr's coat of arms reflects his view of the trade-offs inherent in quantum theory. pioneers of molecular biology, remarked: "It is not to be taken as a forgone conclusion that structure on the molecular scale on the one hand, and integrated function on the other hand are compatible observables."

Given the mechanics of inheritance, and the possibility for environmental change, it should not be surprising to learn that the genomes of organisms are living testimony to compromise. Genes that on average favour reproductive success in one set of conditions often subvert that success under other conditions. The best known human example is the sickle allele at the $\beta$-globin locus, which in single dose protects against falciparum malaria but in double dose usually brings lifethreatening disease. Another trade-off for which there is evidence is that between human longevity and fecundity. In the wake of the announcement that a first draft of the sequence of the human genome has been completed, there have been grand speculations about engineering immortal humans and the like. Have the speculators considered that some of the genotypic 'improvements' they envision might come with substantial, and potentially cryptic, genetic costs?

Moving from a focus on the individual organism to organized human populations - societies - political thought was for centuries dominated by the dream of the perfect society. Various utopians have assumed that there is ultimately a way to organize society so that everyone prospers, all needs are met, and conflict disappears. Fundamental to this conception is the belief that all societal virtues can be pursued in harmony. In contrast, a more recent strain of thought, as elegantly expounded in the writings of Isaiah Berlin, recognizes that similarly desirable virtues, such as freedom and equality or justice and mercy, can inherently conflict. Success in achieving one virtue can come at the cost of diminishing the other.

The list of trade-offs and compromises inherent in various spheres of human activity could probably be extended indefinitely but, alas, here as well one must balance the desire for completeness against the space limitations of publication. The last word deserves to go to Niels Bohr, who offered what may be the most provocative comment pertaining to the fundamental role of limits, trade-offs and compromises. According to his biographer, Abraham Pais, when Bohr was asked to specify what was complementary to truth he replied: clarity.

Neil S. Greenspan is at the Institute of Pathology, Case Western Reserve University, Euclid Avenue, Cleveland, Ohio, 44106-4943, USA. 